\title{
Measurements of a Silicon Photonic ROADM (Project Report CIAN2-3-Y3)
}

\section{SHAYAN MOOKHERJEA}

Department of Electrical and Computer Engineering, University of California, San Diego, MC 0407 La Jolla CA 92093-0407 USA

Email:smookher@ucsd.edu

\section{Introduction and Goals}

A multi-university partnership led by UCSD collaborated with Sandia National Labs in an NSF-funded silicon photonics multi-project wafer (MPW) project in 2013. This was a project "Silicon Photonics and Manufacturing" under the Thrust 2 "Subsystem Integration and Silicon Nanophotonics" of the NSFfunded Center for Integrated Access Networks. The goal of this research was to design, fabricate and test chip-scale opto-electronic devices for use in access and data networks. Designs were submitted for manufacturing in 2013 and diced microchips were returned to the designers for testing in 2014 and thereafter. This is a report of the ROADM +VOA (reconfigurable optical add drop multiplexer + variable optical attenuator) device made using silicon photonics, including passive and doped silicon waveguides and metalization.

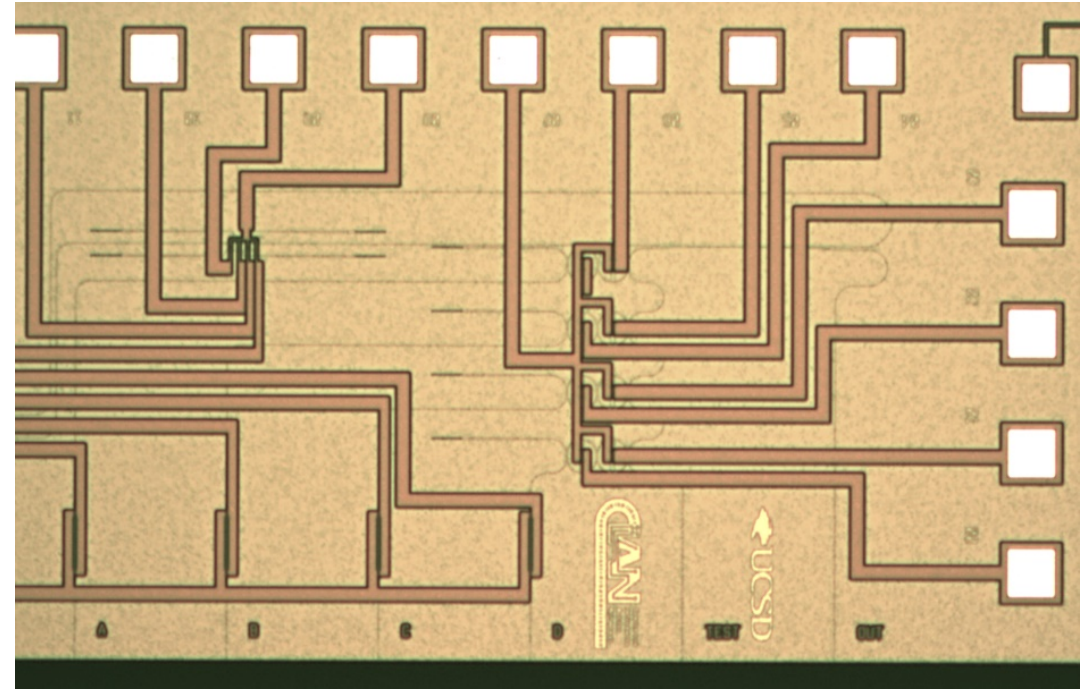

Figure 1 Image of a silicon photonic add-drop multiplexer chip which addresses four WDM channels. A group of four WDM channels (100 GHz spacing) can be added or dropped from the bus waveguide. Variable optical attenuators (VOAs) are also included for each of the four channels as well as the bus input. For coupling waveguides to fibers, an array of edge couplers is used (bottom edge of the diced chip).

\section{Activities}


Figure 1 shows an optical microscope image of the test microchip [1]. Electrical contact pads are situated along the perimeter of the chip and the optical circuitry is in the middle. Edge couplers to fiber are located along the south edge of the chip. A set of four grating couplers is located near the middle of the chip. The purpose of this chip is as follows: a group of four C-band WDM channels (externally modulated with $10 \mathrm{Gbit} / \mathrm{s}$ NRZ data from commercial transceivers at fixed wavelengths) could be added or dropped from a bus waveguide (also called "express channel") which contains up to 24 WDM channels. The adddrop functionality was provided using coupled-microring filters. The channels were tunable using thermo-optic tuning of the microrings. In addition, the power level of each input could be electronically controlled on submicrosecond speeds using a variable optical attenuator (VOA).

This chip was intended for use in a campus ring network (UCSD "MORDIA") using for networking research. This chip was designed to achieve the same functionality as was previously achieved on each node of the testbed using an assembly of conventional off-the-shelf optical components. Off-chip lasers were used for testing. No packaging or driver integration was performed. A bare-die testing workstation was used to measure the performance using both edge-couplers and grating-couplers to fiber arrays and individual fibers.
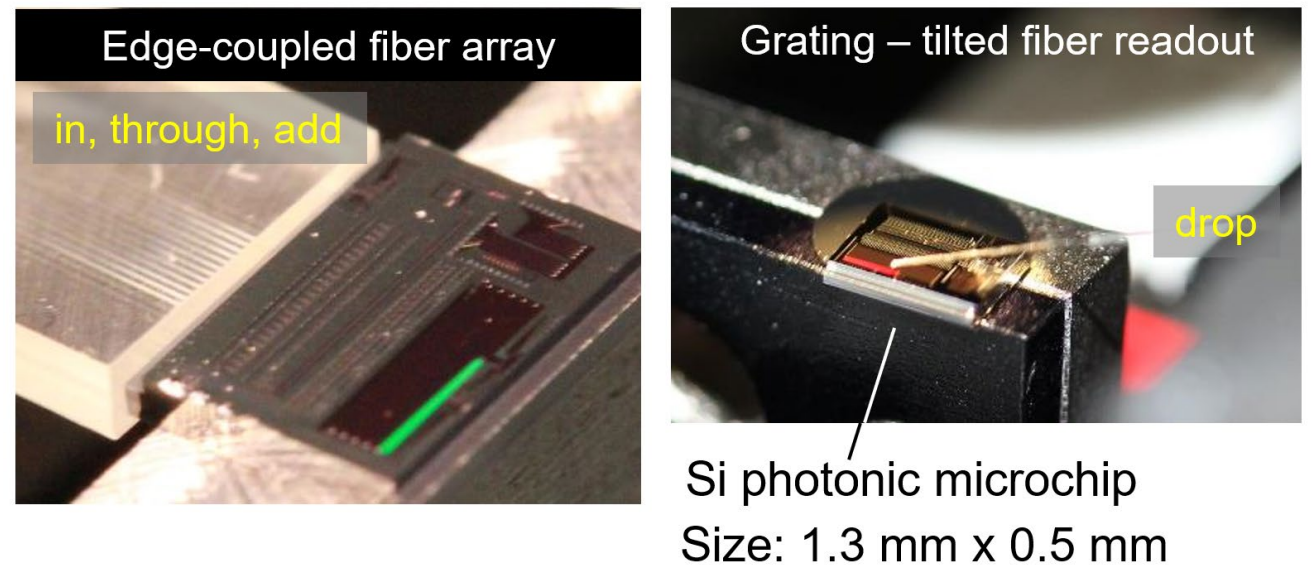

Figure 2 Experimental testing of the unpackaged silicon photonic microchip.

Previously, using a similar experimental workstation, we had tested a silicon photonic chip that were designed and fabricated by Sandia with the same 24channel wavelength division multiplexed (WDM) optical network testbed "MORDIA" at UC San Diego [2]. Standard optical communications transceivers plugged into computer servers were used to generate and receive the data. The wavelength usage plan consisted of six groups of four WDM channels, each modulated at $10 \mathrm{~Gb} / \mathrm{s}$ using the NRZ format. The wavelength usage plan was not exactly periodic, as some channels were left unused. The chip shown in Fig. 1 was designed to be compatible with the same network 
testbed. Therefore, complete tunability across the entire C-band was required of the add and drop components.

Some of the structures in the MPW design used single or coupled silicon microrings. We experimentally studied the dispersion of directional couplers using a microring coupled to a waveguide [3]. An accurate knowledge of dispersion is also necessary for a wide range of integrated photonics devices, such as coupled-microring filters [4], some of which are included in the layout shown in Figure 1.

\section{Progress and Impact}

(a)

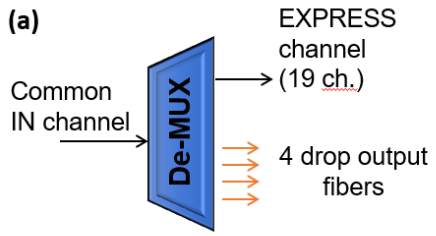

Channels $n, n+1, n+2, n+3$

(group of 4 , spaced by $100 \mathrm{GHz}$ )

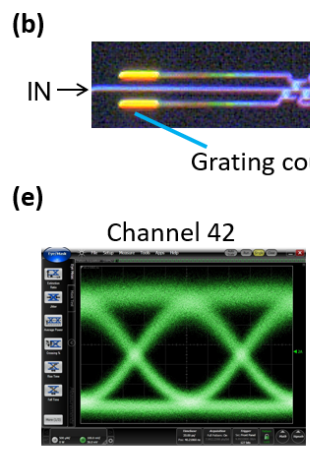

$\mathrm{BER}=3 \mathrm{e}-8$

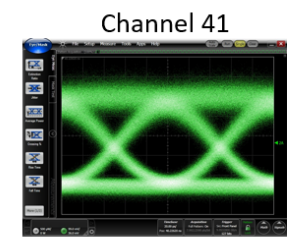

$B E R=4 e-8$ (c)

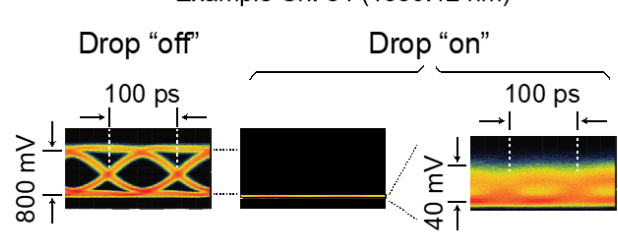

(d)
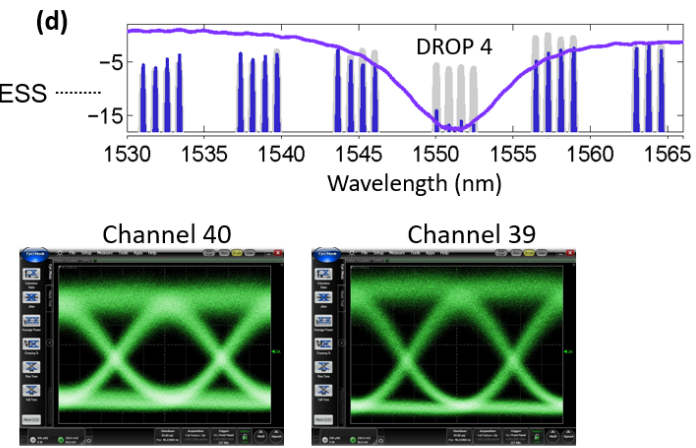

$\mathrm{BER}=7 \mathrm{e}-8$

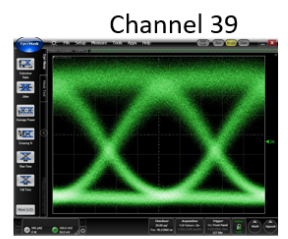

$\mathrm{BER}=1 \mathrm{e}-8$

Figure 3 Drop functionality implemented using coupled-microring filters with thermo-optic tuning in the silicon photonic microchip shown in Figure 1.

Figure 3 shows measurements of the four-channel drop functionality of the microchip. Panel (a) shows the desired functionality, in which a group of 4 channels is dropped from the WDM channel. Four microring resonators were used to achieve the drop functionality. The microrings have a small radius, so that the free spectral range (FSR) is greater than $35 \mathrm{~nm}$ and only one resonance appears in the $\mathrm{C}$-band. An experimental measurement of the before-drop and after-drop transmission spectrum is shown in panel (d), along with a measurement of the overall drop filter response. The filter response was not designed to achieve the perfect brick-wall shape. Each of the four channels was filtered by a thermo-optically tuned coupled-microring filter bank. Nevertheless, a high degree of extinction was achieved. Panel (c) shows the effect on an eye-diagram measurement at the output, showing that the 
amplitude of a dropped channel (as an example, ITU-T DWDM Channel 34 at $1550.12 \mathrm{~nm})$ was reduced by a factor of $50(16 \mathrm{~dB})$ when the filter was tuned to this channel.

Figure 3, panel (e) shows the eye diagrams of four dropped channels measured using the configuration shown in Figure 2, panel (b). Off-chip optical amplification was used to boost the signal levels as the couplers were not optimized, and the chip was not packaged.

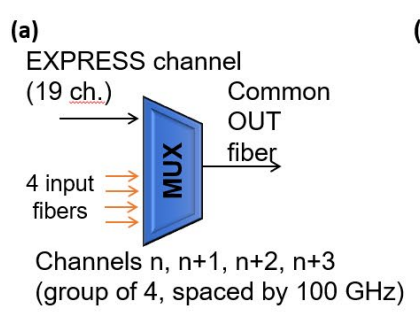

(d)

Example 1: Channels 33 - 36

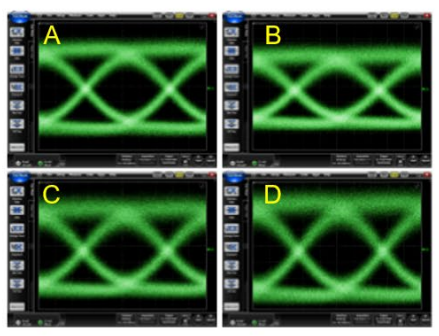

(b)

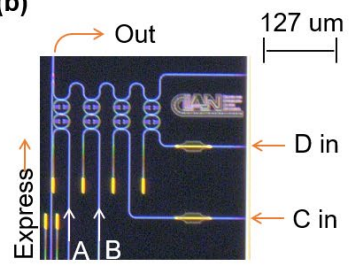

(c)

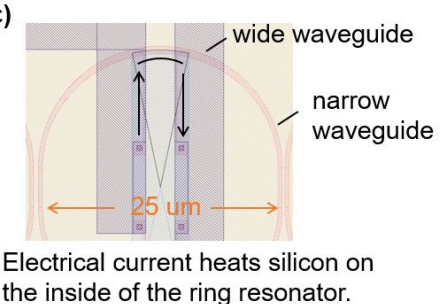

Figure 4 Add functionality implemented using coupled-microring filters with thermo-optic tuning in the silicon photonic microchip shown in Figure 1.

Figure 4 shows measurements of the four-channel add functionality. This is implemented after the drop section, and therefore, larger silicon microrings with a lower FSR can be used. Each channel is added using a coupledmicroring add-drop filter using silicon microring resonators. As shown in panel Fig. 4 panel (c), the rings have a widened waveguide section where doped heaters are incorporated for rapid thermal tuning. Panel (d) shows two examples of a group of four channels being added, with open eye diagrams in each case and a high signal-to-noise ratio exceeding 9.

All the add and drop devices in this design are based on microring resonators with directional couplers to the bus waveguide. When the optical power is increased (typically beyond 10-100 $\mathrm{mW}$ ), silicon microring resonators with a modest Q factor that use directional couplers can exhibit nonlinear behavior, such as bistability [5]. However, these effects are not expected to be manifest at milliwatt power levels.

Coupled microrings were used to achieve an improved filtering response compared to single microring resonators [see Figure 4, panel (b)]. They can 


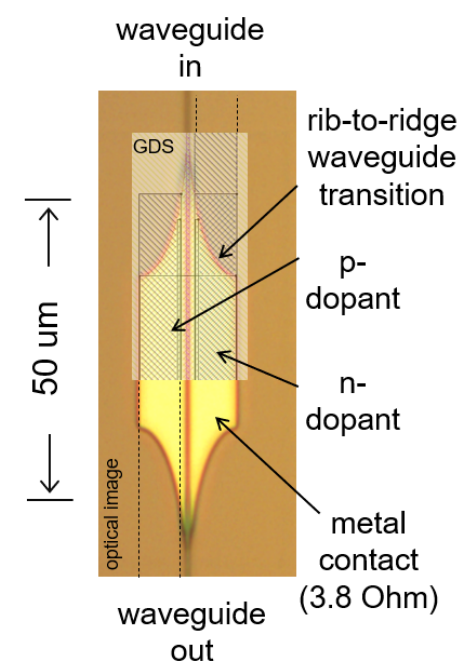

Figure 5 Structure of a Variable Optical Attenuator (VOA) achieve a flat-top transmission passband with steeper sidewalls and less inter-channel crosstalk compared to single ring resonators. Previously, we have developed the theory and design principles of coupled microring resonators, also known as Coupled Resonator Optical Waveguides (CROWs). A tightbinding model of band-center propagation was formulated [6], [7], and theoretical studies of nonlinear propagation were developed on this basis [8], [9]. Studies were performed to examine the regions of high dispersion which may be relevant to signal distortion when filtering wideband signals [10] or all-optically tunable dispersion compensation devices [11]. To help in designing structures in silicon photonics, we have developed an extension of coupled-mode theory which is applicable to directional couplers in high index contrast materials, in coupled-waveguide structures [12] and coupled-resonator structures [13]. An example of a diagnostic measurement and data fitting is shown in Figure 3, showing the very good agreement between realistic models of coupled-microring filters and a measured device. Such diagnostics were essential in achieving good performance from the final fabricated devices.

Five variable optical attenuators (see Figure 5) were included in the design. Each VOA consists of a partially-etched waveguide with $\mathrm{p}$ and $\mathrm{n}$ doped regions near the waveguide core. By injecting electronic carriers into the silicon, the optical transmission through the waveguide can be controlled. The free-carrier absorption effect in silicon is capable of achieving a large variation in optical transmission in a very compact device.

Figure 6 shows an example of equalizing the powers of four channels over a wide dynamic range using VOA's on each individual add channel. VOA's are not optical amplifiers, and can only attenuate the channels whose power level is in excess of the target.

A collaboration with IBM's silicon photonics group (Dr. W. M. J. Green) has provided insights into silicon photonics fabrication and also supported technical collaboration on fabricating structures at wafer-scale using silicon photonics processing [14], [15]. Dr. Ivan B. Divliansky (CREOL, Florida) collaborated with us for the electron-beam lithography of long waveguide structures [16]. CROWs of up to 235 coupled resonators were successfully fabricated and measured, and a good agreement was achieved between experiments and theory [15], [17], [18]. 
Impact on Human Resources: One graduate student in the PI's group worked on this topic as part of their education and training. The Center provided extensive opportunities to interact with researchers and students from other groups and universities as well as with researchers from Sandia.

Spectrum measured on OUT fiber after ADD

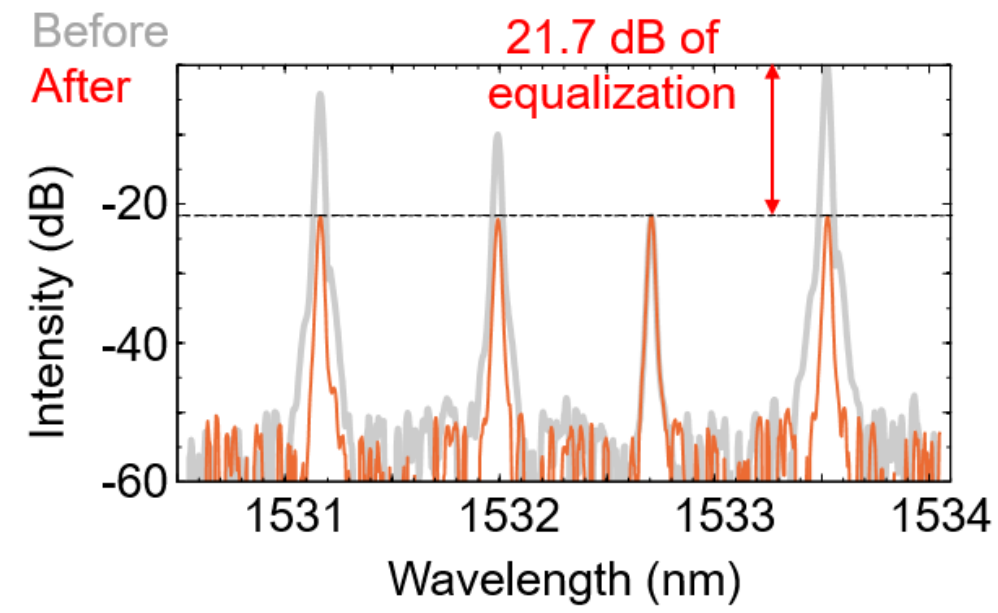

Figure 6 Using VOA's on each ADD input to equalize the power levels of the channels. A large channel-to-channel variation was intentionally introduced to test the range of equalization.

\section{Subsequent Extensions}

CROW structures can have other applications in photonics, such as the generation of entangled photon pairs [19], and developing novel amplifiers and lasers [20]. For example, the resonator-enhanced nonlinearity benefits the generation of entangled photon-pair and heralded single-photon generation using silicon photonics [21]. Our initial measurements of spontaneous fourwave mixing (SFWM) were on correlated photon-pair generation and heralded single-photon generation. An experimental measurement of entanglement was performed later, using a two-photon interferometer constructed to verify timeenergy entanglement [21]. Structures such as VOA's can be useful in improving the amplitude balance between different optical pathways on a microchip circuit. VOA's in silicon photonics can be tuned rapidly (at sub nanosecond speeds) are also useful to protect sensitive detectors from unwanted levels of input optical power, such as the so-called "blinding attacks" on single-photon avalanche diodes.

The filters in the device shown in Figure 2 are based on thermo-optic tunability. Electronic controllability is also of interest in the coupled-microring structure [22]. In contrast to thermal tuning, carrier-driven effects can achieve subnanosecond speeds of varying the optical index and absorption. Even faster effects can be achieved using ferro-electric materials which decouple the 
amplitude and phase effects. For example, optically-transparent thin-film ferro-electric materials bonded to silicon waveguides can be used to achieve phase shifters with more than $100 \mathrm{GHz}$ of bandwidth, as was demonstrated later [23].

Microring resonators are sensitive to disorder. As a way to compensate for defects, we studied potential methods for precisely tuning resonators without heaters. Our method is based on field-induced local oxidation of $\mathrm{Si}$ to $\mathrm{SiO}_{2}$ via a chemical reaction near an electrically-biased conducting atomic-force microscope tip [24]. A single silicon microresonator can be monitored and controlled more easily [25] than a device that consists of many coupled resonators. Infrared imaging can be used to study light propagation through these structures [16], [26] and is a useful tool for finding defects or damaged sites. The microrings with inbuilt heaters in particular were quite easily damaged when tuning was attempted over wide spectral ranges.

\section{Open-Access Reporting Initiative}

PRAISE: This open-access document is provided in support of our PRAISE

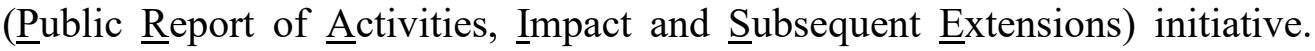
What is it? An open-access document shared with the public which describes the research outcomes of publicly-funded projects. For us, these projects are typically funded by the NSF (National Science Foundation).

\section{References}

[1] R. Aguinaldo et al., "Silicon photonic integrated components for add, drop, and VOA in a 4-channel data-center network," in 2014 IEEE Photonics Conference, San Diego, CA, Dec. 2014, pp. 1-2. doi: 10.1109/IPCon.2014.7092976.

[2] R. Aguinaldo et al., "Wideband silicon-photonic thermo-optic switch in a wavelength-division multiplexed ring network," Opt. Express, vol. 22, no. 7, p. 8205, Apr. 2014, doi: 10.1364/OE.22.008205.

[3] R. Aguinaldo, Yiran Shen, and S. Mookherjea, "Large Dispersion of Silicon Directional Couplers Obtained via Wideband Microring Parametric Characterization," IEEE Photon. Technol. Lett., vol. 24, no. 14, pp. 1242-1244, Jul. 2012, doi: 10.1109/LPT.2012.2198639.

[4] J. R. Ong, R. Kumar, and S. Mookherjea, "Silicon microring-based wavelength converter with integrated pump and signal suppression," Opt. Lett., vol. 39, no. 15, p. 4439, Aug. 2014, doi: 10.1364/OL.39.004439.

[5] S. Mookherjea and M. A. Schneider, "The nonlinear microring add-drop filter," Opt. Express, vol. 16, no. 19, p. 15130, Sep. 2008, doi: 10.1364/OE.16.015130. 
[6] S. Mookherjea and A. Yariv, "Optical pulse propagation in the tightbinding approximation," Opt. Express, vol. 9, no. 2, p. 91, Jul. 2001, doi: 10.1364/OE.9.000091.

[7] S. Mookherjea and A. Yariv, "Pulse propagation in a coupled resonator optical waveguide to all orders of dispersion," Phys. Rev. E, vol. 65, no. 5, p. 056601, Apr. 2002, doi: 10.1103/PhysRevE.65.056601.

[8] S. Mookherjea and A. Yariv, "Kerr-stabilized super-resonant modes in coupled-resonator optical waveguides," Phys. Rev. E, vol. 66, no. 4, p. 046610, Oct. 2002, doi: 10.1103/PhysRevE.66.046610.

[9] S. Mookherjea and A. Yariv, "Optical pulse propagation and holographic storage in a coupled-resonator optical waveguide," Phys. Rev. E, vol. 64, no. 6, p. 066602, Nov. 2001, doi: 10.1103/PhysRevE.64.066602.

[10] S. Mookherjea, D. S. Cohen, and A. Yariv, "Nonlinear dispersion in a coupled-resonator optical waveguide," Opt. Lett., vol. 27, no. 11, p. 933, Jun. 2002, doi: 10.1364/OL.27.000933.

[11] S. Mookherjea, "Using gain to tune the dispersion relation of coupledresonator optical waveguides," IEEE Photon. Technol. Lett., vol. 18, no. 5, pp. 715-717, Mar. 2006, doi: 10.1109/LPT.2006.871144.

[12] M. L. Cooper and S. Mookherjea, "Numerically-assisted coupledmode theory for silicon waveguide couplers and arrayed waveguides," Opt. Express, vol. 17, no. 3, p. 1583, Feb. 2009, doi: 10.1364/OE.17.001583.

[13] S. Mookherjea, "Spectral characteristics of coupled resonators," $J$. Opt. Soc. Am. B, vol. 23, no. 6, p. 1137, Jun. 2006, doi: 10.1364/JOSAB.23.001137.

[14] J. R. Ong et al., "Low-power continuous-wave four-wave mixing in silicon coupled-resonator optical waveguides," Opt. Lett., vol. 36, no. 15, pp. 2964-2966, 2011.

[15] M. L. Cooper et al., "235-ring Coupled-Resonator Optical Waveguides," in Conference on Lasers and Electro-Optics 2010, San Jose, California, 2010, p. CTuHH3. doi: 10.1364/CLEO.2010.CTuHH3.

[16] M. L. Cooper, G. Gupta, J. S. Park, M. A. Schneider, I. B. Divliansky, and S. Mookherjea, "Quantitative infrared imaging of silicon-on-insulator microring resonators," Opt. Lett., vol. 35, no. 5, p. 784, Mar. 2010, doi: 10.1364/OL.35.000784.

[17] S. Mookherjea and M. A. Schneider, "Avoiding bandwidth collapse in long chains of coupled optical microresonators," Opt. Lett., vol. 36, no. 23, p. 4557, Dec. 2011, doi: 10.1364/OL.36.004557.

[18] M. L. Cooper and S. Mookherjea, "Modeling of Multiband Transmission in Long Silicon Coupled-Resonator Optical Waveguides," IEEE Photon. Technol. Lett., vol. 23, no. 13, pp. 872-874, Jul. 2011, doi: 10.1109/LPT.2011.2141657. 
[19] J. R. Ong and S. Mookherjea, "Quantum light generation on a silicon chip using waveguides and resonators," Opt. Express, vol. 21, no. 4, p.

5171, Feb. 2013, doi: 10.1364/OE.21.005171.

[20] S. Mookherjea, "Semiconductor coupled-resonator optical waveguide laser," Appl. Phys. Lett., vol. 84, no. 17, pp. 3265-3267, Apr. 2004, doi: 10.1063/1.1719278.

[21] R. Kumar, M. Savanier, J. R. Ong, and S. Mookherjea, "Entanglement measurement of a coupled silicon microring photon pair source," Opt. Express, vol. 23, no. 15, p. 19318, Jul. 2015, doi: 10.1364/OE.23.019318.

[22] S. Mookherjea, J. R. Ong, X. Luo, and L. Guo-Qiang, "Electronic control of optical Anderson localization modes," Nature Nanotech, vol. 9, no. 5, pp. 365-371, May 2014, doi: 10.1038/nnano.2014.53.

[23] X. Wang, P. O. Weigel, J. Zhao, M. Ruesing, and S. Mookherjea, "Achieving beyond-100-GHz large-signal modulation bandwidth in hybrid silicon photonics Mach Zehnder modulators using thin film lithium niobate," APL Photonics, vol. 4, no. 9, p. 096101, Sep. 2019, doi: 10.1063/1.5115243.

[24] Y. Shen, I. B. Divliansky, D. N. Basov, and S. Mookherjea, "Perfect set-and-forget alignment of silicon photonic resonators and interferometers," in Optical Fiber Communication Conference/National Fiber Optic Engineers Conference 2011, Los Angeles, California, 2011, p. PDPC3. doi: 10.1364/OFC.2011.PDPC3.

[25] M. Savanier, R. Kumar, and S. Mookherjea, "Optimizing photon-pair generation electronically using a $p-i-n$ diode incorporated in a silicon microring resonator," Appl. Phys. Lett., vol. 107, no. 13, p. 131101, Sep. 2015, doi: 10.1063/1.4932047.

[26] S. Mookherjea and H. R. Grant, "High dynamic range microscope infrared imaging of silicon nanophotonic devices," Opt. Lett., vol. 37, no. 22, p. 4705, Nov. 2012, doi: 10.1364/OL.37.004705. 